\title{
Resonance Strength for the Synchrotron Hamiltonian with RF Phase Modulation*
}

\author{
Yunkai Zhang, S.Y. Lee, Indiana University, USA
}

\section{Abstract}

RF phase modulation can be used to measure the beam transfer function in the study of wake field, machine impedance, and the characteristics of collective instabilities. The effect of rf phase modulation can be understood by employing the Hamiltonian analysis of resonance structure. For particle motion with a zero synchronous phase angle, the resonance strength can be analytically evaluated. This paper calculates and tabulates resonance strength functions with non-zero rf synchrotron phase angles. The expansion of the normalized off-momentum coordinates in action-angle phase space coordinates is derived. Our results are compared with that obtained from the canonical perturbation method. Implication of our work in the beam transfer function measurements will be discussed.

\section{INTRODUCTION}

Synchrotron motion of particles in accelerators inevitably experiences perturbation from $\mathrm{rf}$ phase noise, power supply ripple, etc, which can cause rf phase modulation. Meanwhile, rf phase modulation can also be used to measure the beam transfer function in the study of wakefield, machine independence, collective beam instabilities. Therefore, study of rf phase modulation becomes important in some cases. Experiments and theory analysis of rf phase modulation with a zero synchrotron phase angle have been reported in the references $[1,2]$. RF phase modulation can be understood with Hamiltonian analysis of resonance structure. Although analytical analysis for $\mathrm{rf}$ phase modulation with a zero synchrotron phase angle has been obtained, it is difficult to go further with the same approach in the case of non-zero synchrotron phase angle. Then, a numerical method is used to obtain the resonance strength, which is tabulated in the appendix. Comparison with canonical perturbation is given.

This paper goes as follows: Section 2 gives derivation and expansion of the normalized off-momentum coordinate in action-angle phase space coordinates. Section 3 gives comparison with the analytical result in the case of zero phase angle and canonical perturbation with non-zero synchrotron phase angle. Then Conclusion is given in Section 4.

* We wish to thank the support from the US Department of Energy grant DE-FG02-92ER40747 and the National Science Foundation grant PHY-9876773.

\section{HAMILTONIAN RESONANCE STRUCTURE}

The synchrotron Hamiltonian of a single particle in the normalized phase-space coordinates is [3]

$$
H_{0}=\frac{1}{2} P^{2}+\frac{\eta}{|\eta|}\left[\cos \phi-\cos \phi_{s}+\left(\phi-\phi_{s}\right) \sin \phi_{s}\right],
$$

where $P$ is a normalized off-momentum coordinate, $\phi$ is the phase angle, $\phi_{s}$ is the synchronous phase angle, and $\nu_{\mathrm{syn}} \theta$ serves as the independent variable (or time variable). Here $\theta$ is the orbit angle around the synchrotron, and $\nu_{\text {syn }}$ is the synchrotron tune at $\phi_{s}=0$. Without loss of generality, we assume $\eta<0$ hereafter. Since the Hamiltonian $H_{0}$ is "time" independent, it is a constant of motion. Similarly, the action, defined as

$$
J=\frac{1}{2 \pi} \oint P d \phi
$$

is a also a constant of motion. Expressing the Hamiltonian $H_{0}$ as a function of the action $J$, we find the amplitude dependent synchrotron tune becomes $Q_{s}(J)=\partial H_{0} / \partial J$. With the generating function,

$$
F_{2}(\phi, J)=\int_{0}^{\phi} P d \phi,
$$

angle coordinate is

$$
\psi=\frac{\partial F_{2}}{\partial J}=Q_{s}(J) \int_{0}^{\phi} \frac{d \phi}{P} .
$$

In the presence of a harmonic rf phase modulation, the Hamiltonian becomes $H=H_{0}+H_{1}$, where

$$
H_{1}=\nu_{m} a P \cos \left(\nu_{m} \theta+\chi_{0}\right)
$$

is the perturbation term. Here, $\nu_{m}, a$, and $\chi_{0}$ are the ratio of the rf phase modulation tune to $\nu_{\mathrm{syn}}$, the modulation amplitude, and the phase factor.

To study the effect of rf phase modulation on particle motion, We can expand the normalized off-momentum coordinate in the action-angle variables as

$$
P=\sum_{n=-\infty}^{\infty} f_{n}(J) e^{i n \psi}
$$

where

$$
f_{n}(J)=\frac{1}{2 \pi} \int_{0}^{2 \pi} P e^{-i n \psi} d \psi .
$$

Since $P$ is real, we have $f_{-n}=f_{n}^{*}$. Using the identity, $P d \psi=Q_{s} d \phi$, we obtain

$$
f_{n}(J)=\frac{Q_{s}(J)}{2 \pi} \oint e^{-i n \psi} d \phi .
$$


Because $P$ is an odd function, all even harmonics vanish.

When the modulation tune is close to an odd multiple of synchrotron tune, i.e. $\nu_{m} \approx(2 n+1)$ (in unit of $\nu_{\mathrm{syn}}$ ), Using the expansion of phase-space coordinate in action-angle coordinates and neglecting those nonresonance terms, the Hamiltonian becomes

$$
H=H_{0}(J)+\nu_{m} f_{2 n+1} \cos \left((2 n+1) \psi-\nu_{m} \theta-\chi_{0}\right)
$$

. Therefore, after we know the strength of $f_{2 n+1}$, we can analyze the system with rf phase modulation. The table in the appendix gives such resonance strength with different synchrotron phase angle.

\section{COMPARISON}

This section compares the resonance strengths obtained from the exact canonical transformation and perturbation expansion.

\subsection{Analytical method for a zero synchrotron phase angle}

The Hamiltonian with a stationary synchrotron motion is:

$$
H_{0}=\frac{1}{2} P^{2}+2 \sin ^{2} \frac{\phi}{2}
$$

The expansion of $\mathrm{P}$ in Fourier harmonics of $\psi$ is given by [3]

$$
P \approx-\sqrt{2 J} \sin \psi+\frac{(\sqrt{2 J})^{3}}{64} \sin 3 \psi+\cdots
$$

The Figure 1 show the difference between this formula and numerical calculation for the same order, $f_{1}$.

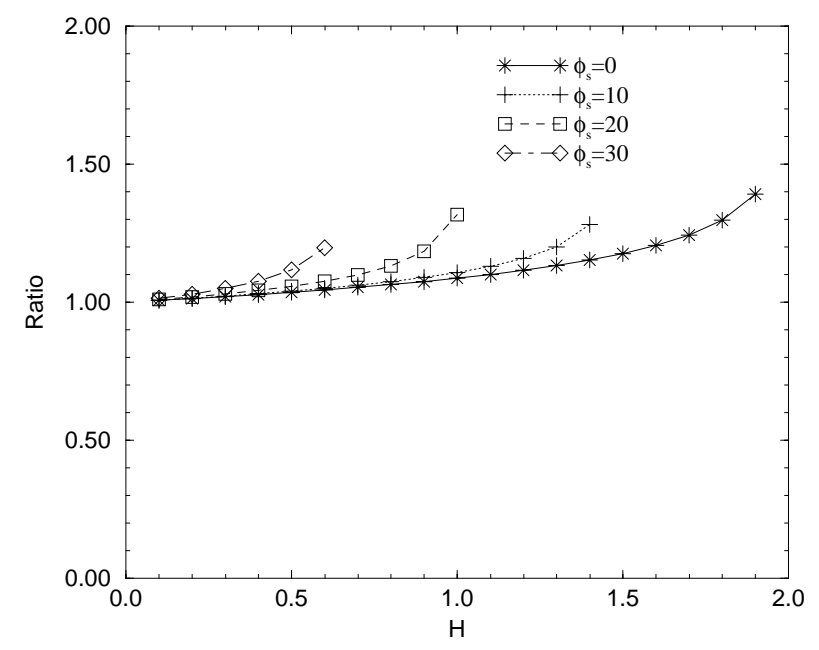

Figure 1: the ratio of the first order resonance strength obtained by analytical and numerical method

\subsection{Canonical perturbation for non-zero syn- chrotron phase angles}

For a Hamiltonian with non-zero synchrotron phase angle, we can expand the phase angle at $\phi_{s}$. Let $\phi=\phi_{s}+\varphi$, the Hamiltonian becomes

$$
H=\frac{1}{2} P^{2}+\frac{\cos \phi_{s}}{2}\left(\varphi^{2}-\frac{1}{3} \tan \phi_{s} \varphi^{3}-\frac{1}{12} \varphi^{4}+\cdots\right)
$$

Choosing the generating function

$$
F_{1}(\phi, \psi)=-\frac{\sqrt{\left|\cos \phi_{s}\right|}}{2} \varphi^{2} \tan \psi
$$

we find the normalized off-momentum coordinate as $P=$ $-\sqrt{2 J} \sin \psi\left|\cos \phi_{s}\right|^{\frac{1}{4}}$, and the new Hamiltonian as

$$
\begin{aligned}
H(J, \psi)= & J \sqrt{\left|\cos \phi_{s}\right|}-\frac{\sqrt{2}}{12}\left|\cos \phi_{s}\right|^{\frac{1}{4}} \tan \phi_{s} J^{\frac{3}{2}} \times \\
& \times(\cos 3 \psi+3 \cos \psi)-\frac{1}{6} J^{2} \cos ^{4} \psi+\cdots
\end{aligned}
$$

Using the generating function,

$$
F_{2}(\psi, I)=\psi I+G_{s}(I) \sin 3 \psi+G_{1}(I) \sin \psi
$$

we get conjugate phase space coordinates as

$$
\begin{aligned}
& J=\frac{\partial F_{2}}{\partial \psi}=I+3 G_{3}(I) \cos 3 \psi+G_{1}(I) \sin \psi \\
& \bar{\psi}=\frac{\partial F_{2}}{\partial \psi}=\psi+3 G_{3}^{\prime}(I) \cos 3 \psi+G_{1}^{\prime}(I) \sin \psi
\end{aligned}
$$

For the first order, we choose

$$
G_{1}(I)=\frac{\sqrt{2}}{4} \frac{\tan \psi_{s} I^{\frac{3}{2}}}{\left|\cos \psi_{s}\right|^{\frac{1}{4}}}, \quad G_{3}(I)=\frac{\sqrt{2}}{36} \frac{\tan \psi_{s} I^{\frac{3}{2}}}{\left|\cos \psi_{s}\right|^{\frac{1}{4}}}
$$

to cancel the term with $J^{\frac{3}{2}}$. Thus the normalized offmomentum becomes

$$
\begin{aligned}
P= & -\left|\cos \psi_{s}\right|^{\frac{1}{4}} \sqrt{2 I}\left(\sin \psi+\frac{1}{4 I} 3 G_{3}(-\sin 2 \psi\right. \\
& +\sin 4 \psi)+\frac{1}{4 I} 3 G_{1}(-\sin 2 \psi) .
\end{aligned}
$$

Comparison of resonance strengths obtained from the canonical perturbation and the exact canonical transformation obtained by numerical integration is shown in Figure 1. The results are tabulated in Tables 1-3.

\section{CONCLUSION}

For analytical analysis in the case of a zero synchrotron phase angle and canonical perturbation in the case of nonzero synchrotron phase angle, they are very good approximation to the first order when the Hamiltonian values are small. When the Hamiltonian value is large, the perturbation expansion can overestimate the resonance strength up to $30 \%$. 
The beam transfer function method measures the response of the beam under the influence of harmonic of phase modulation. The beam detection system measures essentially the dipole moment of the beam distribution. The dipole moment depends on effects of nonlinear detuning, strength of parameter resonances, wake field and potential well distortion, etc. Accurate calculation of known effects of rf potential well provides us a means to study the unknown problems such as the wake field and potential well distribution.

\section{REFERENCES}

[1] M. Ellison et al., Phys. Rev. Lett. 70, 591 (1993).

[2] H. Huang et al., Phys. Rev. E 48, 4678 (1993).

[3] S.Y.Lee, Accelerator Physics, (World Scientific, Singapore, 1999)

Table 1: $\phi_{s}=0^{\circ}$

\begin{tabular}{|c|c|c|c|c|}
\hline $\mathbf{H}$ & $f_{1}$ & $f_{3}$ & $f_{5}$ & $J$ \\
\hline 0.10 & $-0.289 \mathrm{E}-03$ & $0.323 \mathrm{E}-04$ & $-0.299 \mathrm{E}-04$ & 0.101 \\
& $0.446 i$ & $-0.742 \mathrm{E}-03 i$ & $0.461 \mathrm{E}-03 i$ & \\
\hline 0.20 & $-0.383 \mathrm{E}-03$ & $0.438 \mathrm{E}-04$ & $-0.334 \mathrm{E}-04$ & 0.203 \\
& $0.629 i$ & $-0.317 \mathrm{E}-02 i$ & $0.671 \mathrm{E}-03 i$ & \\
\hline 0.30 & $-0.419 \mathrm{E}-03$ & $0.324 \mathrm{E}-04$ & $-0.551 \mathrm{E}-04$ & 0.306 \\
& $0.767 i$ & $-0.666 \mathrm{E}-02 i$ & $0.817 \mathrm{E}-03 i$ & \\
\hline 0.40 & $-0.431 \mathrm{E}-03$ & $0.255 \mathrm{E}-04$ & $-0.314 \mathrm{E}-04$ & 0.411 \\
& $0.883 i$ & $-0.111 \mathrm{E}-01 i$ & $0.990 \mathrm{E}-03 i$ & \\
\hline 0.50 & $-0.433 \mathrm{E}-03$ & $0.200 \mathrm{E}-04$ & $-0.305 \mathrm{E}-04$ & 0.518 \\
& $0.983 i$ & $-0.164 \mathrm{E}-01 i$ & $0.121 \mathrm{E}-02 i$ & \\
\hline 0.60 & $-0.920 \mathrm{E}-03$ & $0.494 \mathrm{E}-04$ & $-0.990 \mathrm{E}-06$ & 0.626 \\
& $1.07 i$ & $-0.230 \mathrm{E}-01 i$ & $0.138 \mathrm{E}-02 i$ & \\
\hline 0.70 & $-0.916 \mathrm{E}-03$ & $0.532 \mathrm{E}-04$ & $0.218 \mathrm{E}-04$ & 0.736 \\
& $1.15 i$ & $-0.303 \mathrm{E}-01 i$ & $0.172 \mathrm{E}-02 i$ & \\
\hline 0.80 & $-0.956 \mathrm{E}-03$ & $0.456 \mathrm{E}-04$ & $0.458 \mathrm{E}-04$ & 0.848 \\
& $1.22 i$ & $-0.387 \mathrm{E}-01 i$ & $0.220 \mathrm{E}-02 i$ & \\
\hline 0.90 & $-0.921 \mathrm{E}-03$ & $0.696 \mathrm{E}-04$ & $0.536 \mathrm{E}-04$ & 0.963 \\
& $1.29 i$ & $-0.483 \mathrm{E}-01 i$ & $0.288 \mathrm{E}-02 i$ & \\
\hline 1.00 & $-0.840 \mathrm{E}-03$ & $0.132 \mathrm{E}-03$ & $0.907 \mathrm{E}-04$ & 1.079 \\
& $1.35 i$ & $-0.592 \mathrm{E}-01 i$ & $0.371 \mathrm{E}-02 i$ & \\
\hline 1.10 & $-0.677 \mathrm{E}-03$ & $0.241 \mathrm{E}-03$ & $0.141 \mathrm{E}-03$ & 1.198 \\
& $1.41 i$ & $-0.717 \mathrm{E}-01 i$ & $0.478 \mathrm{E}-02 i$ & \\
\hline 1.20 & $-0.106 \mathrm{E}-02$ & $0.399 \mathrm{E}-03$ & $0.110 \mathrm{E}-03$ & 1.321 \\
& $1.46 i$ & $-0.861 \mathrm{E}-01 i$ & $0.617 \mathrm{E}-02 i$ & \\
\hline 1.30 & $-0.874 \mathrm{E}-03$ & $0.379 \mathrm{E}-03$ & $-0.391 \mathrm{E}-04$ & 1.447 \\
& $1.50 i$ & $-0.102 i$ & $0.796 \mathrm{E}-02 i$ & \\
\hline 1.40 & $-0.748 \mathrm{E}-03$ & $0.260 \mathrm{E}-03$ & $-0.130 \mathrm{E}-03$ & 1.577 \\
& $1.54 i$ & $-0.122 i$ & $0.103 \mathrm{E}-01 i$ & \\
\hline 1.50 & $-0.115 \mathrm{E}-02$ & $0.318 \mathrm{E}-03$ & $-0.700 \mathrm{E}-04$ & 1.711 \\
& $1.57 i$ & $-0.145 i$ & $0.134 \mathrm{E}-01 i$ & \\
\hline 1.60 & $-0.937 \mathrm{E}-03$ & $0.289 \mathrm{E}-03$ & $-0.178 \mathrm{E}-05$ & 1.852 \\
& $1.60 i$ & $-0.172 i$ & $0.181 \mathrm{E}-01 i$ & \\
\hline & & & & \\
\hline
\end{tabular}

\begin{tabular}{|l|c|c|c|c|}
\hline $\mathbf{H}$ & $f_{1}$ & $f_{3}$ & $f_{5}$ & $J$ \\
\hline 1.70 & $-0.734 \mathrm{E}-03$ & $0.292 \mathrm{E}-03$ & $0.152 \mathrm{E}-04$ & 2.000 \\
& $1.61 i$ & $-0.207 i$ & $0.252 \mathrm{E}-01 i$ & \\
\hline 1.80 & $-0.114 \mathrm{E}-02$ & $0.669 \mathrm{E}-03$ & $-0.193 \mathrm{E}-03$ & 2.157 \\
& $1.60 i$ & $-0.254 i$ & $0.366 \mathrm{E}-01 i$ & \\
\hline 1.90 & $-0.904 \mathrm{E}-03$ & $0.641 \mathrm{E}-03$ & $-0.127 \mathrm{E}-03$ & 2.331 \\
& $1.55 i$ & $-0.325 i$ & $0.594 \mathrm{E}-01 i$ & \\
\hline
\end{tabular}

Table 2: $\phi_{s}=15^{\circ}$

\begin{tabular}{|c|c|c|c|c|}
\hline $\mathbf{H}$ & $f_{1}$ & $f_{3}$ & $f_{5}$ & $J$ \\
\hline 0.10 & $-0.274 \mathrm{E}-03$ & $0.365 \mathrm{E}-04$ & $-0.753 \mathrm{E}-05$ & 0.103 \\
& $0.446 i$ & $-0.688 \mathrm{E}-03 i$ & $0.450 \mathrm{E}-03 i$ & \\
\hline 0.20 & $-0.350 \mathrm{E}-03$ & $0.279 \mathrm{E}-04$ & $-0.277 \mathrm{E}-04$ & 0.207 \\
& $0.628 i$ & $-0.299 \mathrm{E}-02 i$ & $0.629 \mathrm{E}-03 i$ & \\
\hline 0.30 & $-0.747 \mathrm{E}-03$ & $0.441 \mathrm{E}-04$ & $-0.150 \mathrm{E}-04$ & 0.313 \\
& $0.765 i$ & $-0.639 \mathrm{E}-02 i$ & $0.716 \mathrm{E}-03 i$ & \\
\hline 0.40 & $-0.799 \mathrm{E}-03$ & $0.375 \mathrm{E}-04$ & $-0.206 \mathrm{E}-04$ & 0.421 \\
& $0.878 i$ & $-0.104 \mathrm{E}-01 i$ & $0.847 \mathrm{E}-03 i$ & \\
\hline 0.50 & $-0.816 \mathrm{E}-03$ & $0.513 \mathrm{E}-04$ & $-0.855 \mathrm{E}-05$ & 0.530 \\
& $0.976 i$ & $-0.152 \mathrm{E}-01 i$ & $0.987 \mathrm{E}-03 i$ & \\
\hline 0.60 & $-0.819 \mathrm{E}-03$ & $0.603 \mathrm{E}-04$ & $0.188 \mathrm{E}-04$ & 0.643 \\
& $1.06 i$ & $-0.205 \mathrm{E}-01 i$ & $0.115 \mathrm{E}-02 i$ & \\
\hline 0.70 & $-0.732 \mathrm{E}-03$ & $0.125 \mathrm{E}-03$ & $0.691 \mathrm{E}-04$ & 0.758 \\
& $1.14 i$ & $-0.263 \mathrm{E}-01 i$ & $0.132 \mathrm{E}-02 i$ & \\
\hline 0.80 & $-0.593 \mathrm{E}-03$ & $0.926 \mathrm{E}-04$ & $0.289 \mathrm{E}-04$ & 0.876 \\
& $1.20 i$ & $-0.322 \mathrm{E}-01 i$ & $0.140 \mathrm{E}-02 i$ & \\
\hline 0.90 & $-0.977 \mathrm{E}-03$ & $0.111 \mathrm{E}-03$ & $0.456 \mathrm{E}-04$ & 0.998 \\
& $1.26 i$ & $-0.379 \mathrm{E}-01 i$ & $0.110 \mathrm{E}-02 i$ & \\
\hline 1.00 & $-0.875 \mathrm{E}-03$ & $0.717 \mathrm{E}-04$ & $0.620 \mathrm{E}-04$ & 1.125 \\
& $1.30 i$ & $-0.413 \mathrm{E}-01 i$ & $0.337 \mathrm{E}-03 i$ & \\
\hline 1.10 & $-0.597 \mathrm{E}-03$ & $0.127 \mathrm{E}-03$ & $0.717 \mathrm{E}-04$ & 1.258 \\
& $1.31 i$ & $-0.376 \mathrm{E}-01 i$ & $-0.218 \mathrm{E}-02 i$ & \\
\hline 1.20 & $-0.530 \mathrm{E}-03$ & $0.842 \mathrm{E}-04$ & $0.816 \mathrm{E}-04$ & 1.403 \\
& $1.27 i$ & $-0.863 \mathrm{E}-03 i$ & $-0.127 \mathrm{E}-01 i$ & \\
\hline
\end{tabular}

Table 3: $\phi_{s}=30^{\circ}$

\begin{tabular}{|l|c|c|c|c|}
\hline $\mathbf{H}$ & $f_{1}$ & $f_{3}$ & $f_{5}$ & $J$ \\
\hline 0.10 & $-0.407 \mathrm{E}-03$ & $0.121 \mathrm{E}-04$ & $-0.124 \mathrm{E}-04$ & 0.109 \\
& $0.444 i$ & $-0.448 \mathrm{E}-03 i$ & $0.388 \mathrm{E}-03 i$ & \\
\hline 0.20 & $-0.463 \mathrm{E}-03$ & $0.398 \mathrm{E}-04$ & $0.369 \mathrm{E}-05$ & 0.220 \\
& $0.622 i$ & $-0.192 \mathrm{E}-02 i$ & $0.494 \mathrm{E}-03 i$ & \\
\hline 0.30 & $-0.473 \mathrm{E}-03$ & $0.674 \mathrm{E}-04$ & $0.365 \mathrm{E}-04$ & 0.336 \\
& $0.753 i$ & $-0.333 \mathrm{E}-02 i$ & $0.565 \mathrm{E}-03 i$ & \\
\hline 0.40 & $-0.407 \mathrm{E}-03$ & $0.332 \mathrm{E}-04$ & $-0.409 \mathrm{E}-05$ & 0.455 \\
& $0.855 i$ & $-0.367 \mathrm{E}-02 i$ & $0.395 \mathrm{E}-03 i$ & \\
\hline 0.50 & $-0.683 \mathrm{E}-03$ & $-0.497 \mathrm{E}-06$ & $0.200 \mathrm{E}-04$ & 0.580 \\
& $0.930 i$ & $-0.611 \mathrm{E}-03 i$ & $-0.310 \mathrm{E}-03 i$ & \\
\hline 0.60 & $-0.359 \mathrm{E}-03$ & $0.258 \mathrm{E}-04$ & $0.823 \mathrm{E}-04$ & 0.715 \\
& $0.964 i$ & $0.163 \mathrm{E}-01 i$ & $-0.249 \mathrm{E}-02 i$ & \\
\hline
\end{tabular}

